\title{
A COMPARISON BETWEEN DEHYDRATION FROM SALT LOSS AND FROM WATER DEPRIVATION
}

\author{
By JOSEPH W. NADAL, SVEND PEDERSEN, AND WALTER G. MADDOCK \\ (From the Department of Surgery of the University of Michigan, Ann Arbor) ${ }^{1}$
}

(Received for publication July 7, 1941)

In 1935 Kerpel-Fronius (1) demonstrated in rabbits that there are two distinct types of dehydration, depending upon whether the condition is associated with (1) a primary loss of salt or (2) a shortage of water not accompanied by a corresponding loss of salt. He pointed out that the former is characterized by circulatory disturbances, while the latter is characterized by thirst. In a later paper (2) Kerpel-Fronius referred to the two conditions as "Durstexsikkose und Salzmangelexsikkose" and emphasized the fact that in animals dehydrated by loss of salt the principal loss of fluid is from the extracellular portion, including the blood, whereas in thirsting animals the water loss is distributed among all fluid compartments of the body, including the large intracellular portion.

It is our impression that most physicians, including ourselves, have not been sufficiently aware of this important distinction and its therapeutic implications. The present report concerns itself with a study of these two types of dehydration in normal human subjects. The first two experiments (IA and IB) deal with abnormal loss of salt while the water intake is adequate. The other two experiments (IIA and IIB) deal with water deprivation with no abnormal loss of salt.

\section{PROCEDURE}

Experiment IA. Abnormal loss of salt; water intake adequate. Subject W. M., a healthy male, aged 26 , was weighed and placed upon a salt-poor diet (containing approximately 1 gram of $\mathrm{NaCl}$ per day) for 6 days. After this preliminary period a Miller-Abbott tube (3) was passed to the proximal jejunum, the position of the tube was verified by $x$-ray examination, and constant suction was applied for 4 days. During this 4-day period no food was allowed, but water was administered daily, either orally or in the form of intravenous 5 per cent dextrose in distilled water, in amounts sufficient to insure a normal urinary output. Also, the tube was irrigated frequently with measured amounts of tap water which

1 Aided by a grant from the Horace $H$. Rackham School of Graduate Studies. were figured in as part of the total oral intake. After removal of the Miller-Abbott tube, the subject was allowed salt-free water orally and intravenously for a period of 24 hours. On the following day he was given 3675 cc. of Ringer's solution (containing 33.1 grams of $\mathrm{NaCl}$ ) intravenously. On the 2 final days he was allowed water ad libitum and a salt-poor diet.

Each morning after voiding the subject was weighed. Daily measurements were made of the urine volume and of the losses of sodium and chloride in the urine and from the jejunal drainage. ${ }^{2}$ Frequent determinations ${ }^{2}$ were also made of the hematocrit, plasma proteins, serum sodium, plasma chlorides, plasma carbon dioxide combining power, and of the arterial blood pressure. The subject was watched closely for any signs of dehydration.

Experiment IB. Abnormal loss of salt; water intake adequate. Subject E. B., aged 21, was a normal male except for the presence of an orthostatic albuminuria. The procedure was identical to that in Experiment IA with the following exceptions. The Miller-Abbott tube suction was continued for 5 days instead of 4 . Distilled water was used in place of tap water throughout the experiment. The salt-poor diet was resumed as soon as the Miller-Abbott tube had been removed. Three thousand cubic centimeters of Ringer's solution (containing 27 grams of $\mathrm{NaCl}$ ) were administered on the second day following removal of the tube.

Experiment IIA. Water deprivation; no abnormal loss of salt. Subject E. B. at the conclusion of Experiment IB was studied further. He continued on a salt-poor diet for 2 days and had water ad libitum. Then the dehydration period began and all fluids were eliminated for 4 days. On the 5 th day his mouth was so dry he could not eat, so $600 \mathrm{cc}$. of distilled water were allowed. During the recovery period distilled water was allowed ad libitum, the diet remaining salt-poor as before. Blood studies and other measurements were carried out as in Experiments IA and IB. Also several determinations were made of the blood non-protein nitrogen.

Experiment IIB. Water deprivation by means of total

2 The chemical determinations were run in duplicate and standard methods were used throughout as follows: Sodium: Butler and Tuthill (4). Potassium: Shohl and Bennett (5). Chloride: Wilson and Ball (6). Nitrogen: Macro-Kjeldahl. Total urine solids: Shackell (7). Carbon dioxide combining power: Van Slyke (8). The plasma protein estimations were based upon plasma specific gravities according to Weech's formula (9), the specific gravity determinations being made by the Barbour and Hamilton falling drop method (10). 
fasting. This experiment, though somewhat similar to Experiment IIA, differed from it chiefly in that the dehydration period consisted of a total fast. The subject. T. G., a normal male aged 23 , after having been placed upon a salt-poor diet for 4 days, was persuaded to go without food or water for 3 days. During the subsequent 3 days he still received no food but was allowed distilled water ad libitum. In fact, he was requested to drink even more than he desired, and on the last of these 3 days (day 10) he was given, in addition, 4.8 grams of potassium as a dilute neutral solution of potassium salts $\left(\mathrm{K}_{2} \mathrm{HPO}_{4}\right.$ and $\left.\mathrm{KH}_{2} \mathrm{PO}_{4}\right)$. On the next 2 days (days 11 and 12) he was given a submaintenance diet consisting of 2 liters of whole cow's milk per day. On the 12th day he was given, in addition to the milk, 9 grams of sodium chloride.

Every morning after voiding the subject was carefully weighed on a balance accurate to 2 grams. Daily determinations were made of the urine volume, urine specific gravity, urine solids, and urinary excretions of sodium, potassium, chloride, and nitrogen. The same blood studies were carried out as in the other three experiments. During the difficult fasting period the subject was attended both day and night and seemed to cooperate in every particular.

\section{RESULTS}

In Experiment IA, the data of which are shown in Table I and Figure 1, the body weight de- creased by $4.3 \mathrm{kgm}$. during the 4 days of the Miller-Abbott suction. During this period 8.4 grams of $\mathrm{Na}$ and 13.9 grams of $\mathrm{Cl}$, equivalent to about 22 grams of $\mathrm{NaCl}$, were lost from the body, mostly through the tube, only a very small amount being excreted in the urine. The urine output averaged $1430 \mathrm{cc}$. per day. The hematocrit and plasma protein concentration showed a gradual and continuous increase. The serum $\mathrm{Na}$ concentration dropped from 308 to $274 \mathrm{mgm}$. per cent, a decrease of about 11 per cent, while the plasma $\mathrm{Cl}$ concentration fell from 568 to $464 \mathrm{mgm}$. per cent, a decrease of about 18 per cent. The $\mathrm{CO}_{2}$ combining power showed little change. Towards the end of the dehydration period, the subject became weak and apathetic and the blood pressure fell to $85 \mathrm{~mm}$. $\mathrm{Hg}$ systolic. He became faint on assuming the upright posture and preferred to lie flat in bed without a pillow. There was pronounced anorexia. There was no thirst.

During the 24-hour period immediately after removal of the tube (day 11) the situation was essentially unchanged. The hematocrit and plasma protein concentration remained high while the

TABLE I

Data on Experiment IA. Subject W. M. Dehydration by means of constant jejunal suction with Miller-Abbott tube; water intake adequate

\begin{tabular}{|c|c|c|c|c|c|c|c|c|c|c|c|c|c|c|c|}
\hline \multirow[b]{2}{*}{ Days } & \multirow[b]{2}{*}{$\begin{array}{l}\text { Body } \\
\text { weight }\end{array}$} & \multicolumn{2}{|c|}{ Fluid intake } & \multicolumn{3}{|c|}{ Suction } & \multicolumn{3}{|c|}{ Urine } & \multicolumn{5}{|c|}{ Blood } & \multirow[b]{2}{*}{ Remarks } \\
\hline & & Mouth & $\begin{array}{l}\text { Intra- } \\
\text { venous }\end{array}$ & Amount & $\mathbf{N a}$ & $\mathrm{Cl}$ & Amount & $\mathbf{N a}$ & $\mathrm{Cl}$ & $\begin{array}{l}\text { Hem- } \\
\text { ato- } \\
\text { crit }\end{array}$ & $\begin{array}{r}\text { Plasma } \\
\text { protein }\end{array}$ & $\underset{\mathbf{N a}}{\text { Serum }}$ & $\underset{\mathrm{Cl}}{\text { Plasma }}$ & $\begin{array}{c}\text { Plasma } \\
\text { CO2. } \\
\text { C.P. }\end{array}$ & \\
\hline 0 & $\begin{array}{l}\mathrm{kgm} . \\
70.1\end{array}$ & $c c$. & $\begin{array}{c}c c . \\
5 \% \\
\text { dextrose }\end{array}$ & $c c$. & grams & grams & $c c$. & grams & grams & $\begin{array}{c}\text { per } \\
\text { cent } \\
47.2\end{array}$ & $\begin{array}{c}\text { grams } \\
\text { per } \\
\text { cent } \\
7.2\end{array}$ & 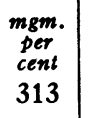 & $\begin{array}{c}\text { mgm. } \\
\text { per cent } \\
(\text { as NaCl }) \\
566\end{array}$ & $\begin{array}{c}\text { volume } \\
\text { per } \\
\text { cent } \\
47\end{array}$ & \\
\hline $\begin{array}{l}1 \\
2 \\
3 \\
4 \\
5 \\
6\end{array}$ & \begin{tabular}{l|}
69.1 \\
69.0 \\
69.2 \\
68.7 \\
68.7 \\
68.9
\end{tabular} & & & & & & $\begin{array}{r}1520 \\
1050 \\
840 \\
800 \\
1150 \\
1450\end{array}$ & \begin{tabular}{|l|}
5.43 \\
2.32 \\
2.61 \\
2.50 \\
0.89 \\
0.45
\end{tabular} & $\begin{array}{l}8.78 \\
3.35 \\
3.68 \\
3.59 \\
1.44 \\
0.86\end{array}$ & 50.5 & 7.2 & 308 & 568 & 47 & Salt-poor diet. \\
\hline $\begin{array}{r}7 \\
8 \\
9 \\
10\end{array}$ & \begin{tabular}{|l|}
66.5 \\
66.0 \\
65.4 \\
64.6 \\
\end{tabular} & $\begin{array}{l}1500 \\
2000 \\
2750 \\
1500\end{array}$ & $\begin{array}{r}0 \\
1900 \\
950 \\
1850\end{array}$ & $\begin{array}{l}1460 \\
1540 \\
2230 \\
1040\end{array}$ & $\begin{array}{l}1.95 \\
2.40 \\
2.14 \\
1.05\end{array}$ & $\begin{array}{l}3.42 \\
3.70 \\
3.49 \\
1.89\end{array}$ & $\begin{array}{r}1380 \\
1540 \\
840 \\
1960\end{array}$ & \begin{tabular}{l|}
0.49 \\
0.17 \\
0.09 \\
0.04
\end{tabular} & \begin{tabular}{|l|}
1.22 \\
0.12 \\
0.07 \\
0.11 \\
\end{tabular} & $\begin{array}{l}53.3 \\
59.2 \\
59.3 \\
60.2\end{array}$ & $\begin{array}{l}8.1 \\
8.8 \\
8.5 \\
8.6\end{array}$ & $\begin{array}{l}271 \\
284 \\
288 \\
274\end{array}$ & $\begin{array}{l}553 \\
512 \\
477 \\
479\end{array}$ & $\begin{array}{l}31 \\
41 \\
50 \\
38\end{array}$ & $\begin{array}{l}\text { Miller-Abbott } \\
\text { tube suction. } \\
\text { No diet. }\end{array}$ \\
\hline 11 & 64.3 & 250 & 2000 & & & & 1410 & 0.02 & 0.13 & 60.0 & 8.2 & 282 & 464 & 47 & $\begin{array}{l}\text { Salt-free water } \\
\text { given. }\end{array}$ \\
\hline 12 & 66.4 & 0 & $3675^{*}$ & & & & 810 & 0.40 & 0.44 & 42.4 & 6.1 & 298 & 574 & 41 & $\begin{array}{l}\text { Ringer's solu- } \\
\text { tion given. }\end{array}$ \\
\hline $\begin{array}{l}13 \\
14\end{array}$ & \begin{tabular}{l|}
65.7 \\
65.9
\end{tabular} & $\begin{array}{c}2000 \\
\text { Ad lib. }\end{array}$ & $\begin{array}{l}\mathbf{0} \\
\mathbf{0}\end{array}$ & & & & $\begin{array}{l}1370 \\
1550\end{array}$ & $\begin{array}{l}1.59 \\
2.00\end{array}$ & \begin{tabular}{|l|}
1.91 \\
2.10
\end{tabular} & $\begin{array}{l}42.2 \\
44.8\end{array}$ & $\begin{array}{l}6.5 \\
6.8\end{array}$ & $\begin{array}{l}308 \\
325\end{array}$ & $\begin{array}{l}568 \\
569\end{array}$ & $\begin{array}{l}41 \\
45\end{array}$ & $\begin{array}{l}\text { Salt-poor diet } \\
\text { resumed. }\end{array}$ \\
\hline
\end{tabular}

* Ringer's solution. 


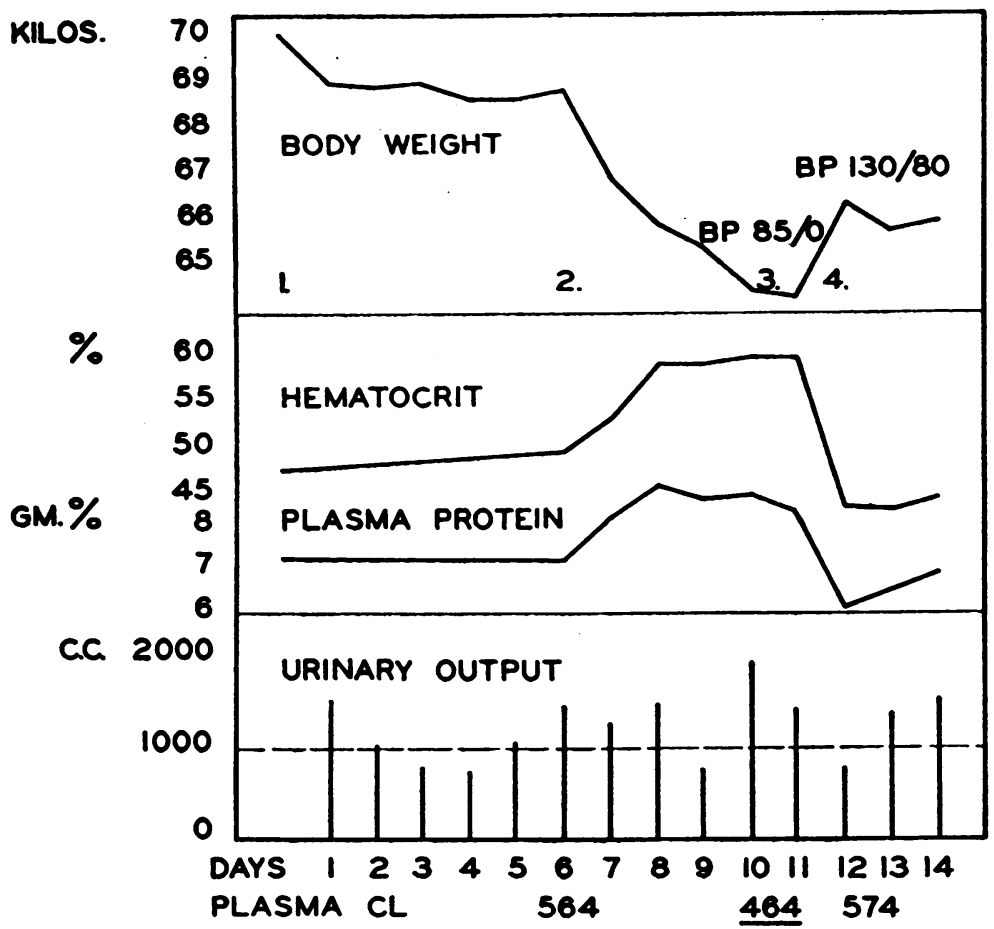

I. SALT POOR DIET

2. MILLER-ABBOTT. TUBE

NA LOSS 8.4 GM.(21.0)

CL LOSS $13.9 \mathrm{GM}$ (23.1)

3. TUBE OUT

SALT FREE WATER GIVEN

4. RINGER'S SOLUTION

3675 C.C. 'I.V.

Fig. 1. Graphic Presentation of Experiment iA. Subject W. M. Dehydration by Means of Constant Jejunal Suction With Miller-Abbott Tube; Water Intake Adequate

serum $\mathrm{Na}$, plasma $\mathrm{Cl}$, and the blood pressure remained low. The weakness, anorexia, apathy, and orthostatic fainting continued.

As soon as the Ringer's solution was administered, on the 12th day, there was a prompt improvement in the subject's condition. The blood became diluted, the serum $\mathrm{Na}$ and plasma $\mathrm{Cl}$ returned to normal, the blood pressure rose to normal, and the disagreeable symptoms vanished.

At the end of the recovery period, the body weight was about $3 \mathrm{kgm}$. less than at the beginning of the experiment, undoubtedly the result of 6 days of fasting. The final values for hematocrit and plasma protein were lower than the initial values, probably due to the fact that during the course of the experiment a total of about $400 \mathrm{cc}$. of blood was taken for the various blood tests.

Experiment IB was essentially the same as IA and yielded similar results. As shown by the data in Table II and Figure 2, there occurred a loss in weight, a rise in hematocrit and a drop in blood pressure. Fainting, weakness, apathy and anorexia were pronounced. There was no thirst; in fact, there seemed to be an aversion to water.
It will be noted that the plasma protein values did not rise so noticeably in Experiment IB as in Experiment IA and this might be related to the orthostatic albuminuria of the IB subject. The administration of Ringer's solution restored the subject to normal.

The results in Experiments IIA and IIB stand out in marked contrast to those in IA and IB. In Experiment IIA there was no abnormal loss of salt and dehydration was brought about by water deprivation. The data given in Table III and Figure 3 show a loss in body weight of 2.6 $\mathrm{kgm}$. during the period of water deprivation. There was no significant change in hematocrit, serum $\mathrm{Na}$, or plasma $\mathrm{CO}_{2}$ combining power. The plasma $\mathrm{Cl}$ rose from 554 to $584 \mathrm{mgm}$. per $100 \mathrm{cc}$. The daily urinary output averaged $614 \mathrm{cc}$. The excretion of $\mathrm{Na}$ and $\mathrm{Cl}$ increased slightly during the period of water deprivation, but remained low nevertheless. The blood non-protein nitrogen rose from 33 to $48 \mathrm{mgm}$. per cent. Thirst became acute and the mouth and throat became very dry. The voice became weak and high in pitch. It will be recalled that on the last day of the dehy- 
TABLE II

Data on Experiment IB. Subject E. B. Dehydration by means of constant jejunal suction with Miller-Abbott tube; water intake adequate

\begin{tabular}{|c|c|c|c|c|c|c|c|c|c|c|c|c|c|c|c|}
\hline \multirow[b]{2}{*}{ Days } & \multirow{2}{*}{$\begin{array}{c}\text { Body } \\
\text { weight }\end{array}$} & \multicolumn{2}{|c|}{ Fluid intake } & \multicolumn{3}{|c|}{ Suction } & \multicolumn{3}{|c|}{ Urine } & \multicolumn{5}{|c|}{ Blood } & \multirow[b]{2}{*}{ Remarks } \\
\hline & & Mouth & $\begin{array}{l}\text { Intra- } \\
\text { venous }\end{array}$ & Amount & $\mathrm{Na}$ & $\mathrm{Cl}$ & Amount & $\mathrm{Na}$ & $\mathrm{Cl}$ & $\begin{array}{l}\text { Hem- } \\
\text { ato- } \\
\text { crit }\end{array}$ & $\begin{array}{l}\text { Plasma } \\
\text { protein }\end{array}$ & $\underset{\mathbf{N a}}{\text { Serum }}$ & $\underset{\mathrm{Cl}}{\text { Plasma }}$ & $\mid \begin{array}{c}\text { Plasma } \\
\mathrm{CO}_{2} \\
\text { C.P. }\end{array}$ & \\
\hline 0 & $\begin{array}{l}k g m . \\
59.7\end{array}$ & $c c$. & $\begin{array}{c}c c . \\
5 \% \\
\text { dextrose }\end{array}$ & $c c$. & grams & grams & $c c$. & grams & grams & $\begin{array}{c}\text { per } \\
\text { cent } \\
44.3\end{array}$ & 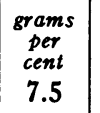 & $\begin{array}{c}m g m . \\
\text { per } \\
\text { cent } \\
292\end{array}$ & \begin{tabular}{|} 
mgm. \\
per cent \\
(as NaCl) \\
574
\end{tabular} & \begin{tabular}{|c|} 
volume \\
per \\
cent \\
49
\end{tabular} & \\
\hline $\begin{array}{l}1 \\
2 \\
3 \\
4 \\
5 \\
6\end{array}$ & $\begin{array}{l}\mathbf{5 9 . 0} \\
\mathbf{5 8 . 8} \\
\mathbf{5 8 . 6} \\
\mathbf{5 8 . 5} \\
\mathbf{5 8 . 8} \\
\mathbf{5 8 . 7}\end{array}$ & & & & & & $\begin{array}{r}995 \\
1130 \\
1000 \\
1110 \\
870 \\
1170\end{array}$ & $\begin{array}{l}1.87 \\
0.88 \\
0.64 \\
0.45 \\
0.30 \\
0.35\end{array}$ & \begin{tabular}{|l}
3.54 \\
1.68 \\
1.22 \\
1.04 \\
0.56 \\
0.48
\end{tabular} & $\begin{array}{l}45.3 \\
45.6 \\
44.6\end{array}$ & $\begin{array}{l}7.5 \\
7.7 \\
7.5\end{array}$ & $\begin{array}{l}292 \\
297 \\
302\end{array}$ & $\begin{array}{l}561 \\
553 \\
554\end{array}$ & $\begin{array}{l}51 \\
55 \\
58\end{array}$ & Salt-poor diet. \\
\hline $\begin{array}{r}7 \\
8 \\
9 \\
10 \\
11\end{array}$ & $\begin{array}{l}\mathbf{5 7 . 0} \\
56.3 \\
55.4 \\
54.3 \\
53.9\end{array}$ & $\begin{array}{l}4000 \\
2600 \\
3200 \\
2500 \\
2500\end{array}$ & $\begin{array}{r}0 \\
2000 \\
0 \\
0 \\
0\end{array}$ & $\begin{array}{l}4300 \\
1090 \\
1730 \\
1560 \\
1270\end{array}$ & $\begin{array}{l}2.18 \\
1.47 \\
2.16 \\
1.87 \\
1.00\end{array}$ & $\begin{array}{l}4.59 \\
2.28 \\
3.39 \\
3.06 \\
1.69\end{array}$ & $\begin{array}{r}540 \\
2980 \\
1580 \\
1080 \\
750\end{array}$ & $\begin{array}{l}0.10 \\
0.06 \\
0.01 \\
0.04\end{array}$ & $\begin{array}{l}0.18 \\
0.16 \\
0.08 \\
0.06 \\
0.02\end{array}$ & $\begin{array}{l}46.6 \\
48.0 \\
51.7 \\
53.8 \\
57.1\end{array}$ & $\begin{array}{l}7.5 \\
7.6 \\
7.5 \\
7.7 \\
7.8\end{array}$ & $\begin{array}{l}301 \\
291 \\
299 \\
274 \\
270\end{array}$ & $\begin{array}{l}531 \\
526 \\
507 \\
484 \\
467\end{array}$ & $\begin{array}{l}51 \\
53 \\
50 \\
48 \\
51\end{array}$ & $\begin{array}{l}\text { Miller-Abbott } \\
\text { tube suction. } \\
\text { No diet. }\end{array}$ \\
\hline 12 & 54.0 & & 0 & & & & 780 & 0.005 & 0.04 & 57.0 & 7.9 & 269 & 465 & 61 & $\begin{array}{l}\text { Salt-poor diet } \\
\text { resumed. }\end{array}$ \\
\hline 13 & 57.0 & & $3000^{*}$ & & & & 1430 & 0.03 & 0.23 & 39.0 & 6.0 & 307 & 554 & 57 & $\begin{array}{l}\text { Ringer's solu- } \\
\text { tion given. }\end{array}$ \\
\hline $\begin{array}{l}14 \\
15\end{array}$ & $\begin{array}{l}56.9 \\
57.3\end{array}$ & & & & & & $\begin{array}{l}1310 \\
1360\end{array}$ & $\begin{array}{l}0.14 \\
0.45\end{array}$ & $\begin{array}{l}0.35 \\
0.54\end{array}$ & 40.0 & 6.4 & 299 & 553 & 55 & $\begin{array}{l}\text { Salt-poor diet } \\
\text { continued. }\end{array}$ \\
\hline
\end{tabular}

* Ringer's solution.

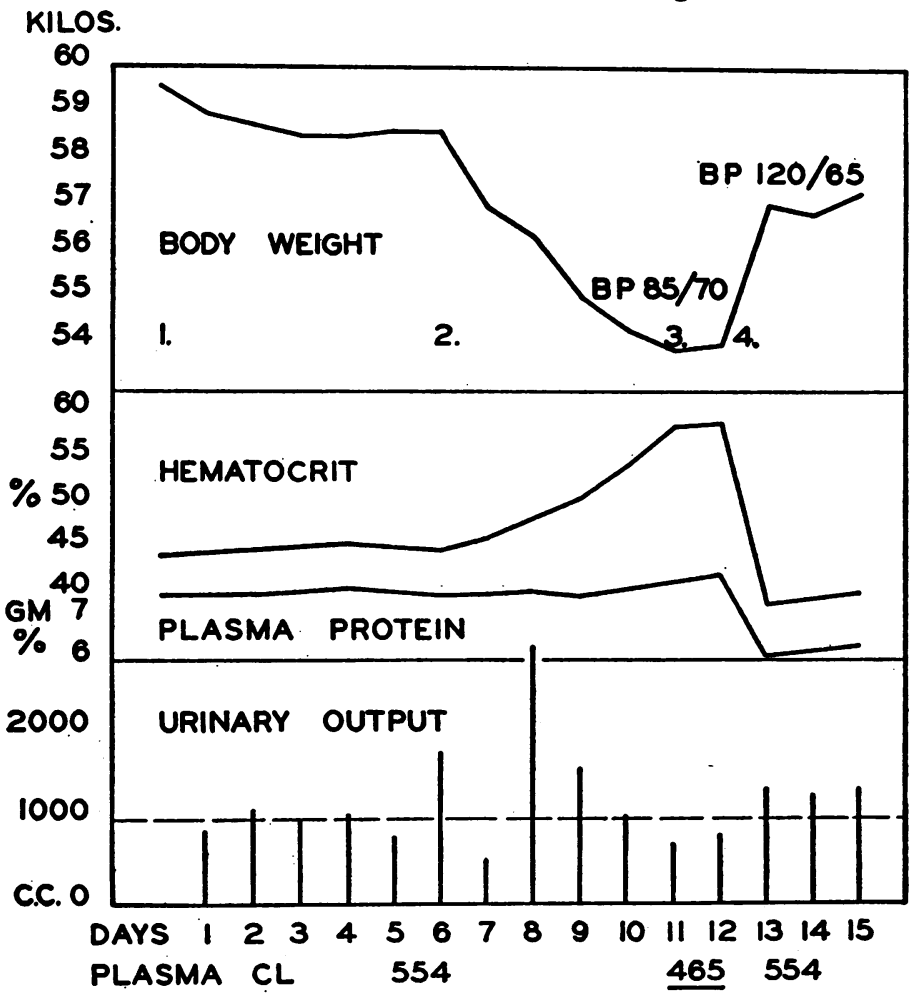

I. SALT POOR DIET

2. MILLER-ABBOT TUBE

NA LOSS 9.0 GM. $|22.5|$

CL LOSS 15.6 GM. (25.7)

3. TUBE OUT

SALT FREE WATER GIVEN

4. RINGER'S SOLUTION

30.00 C.C. I.V.

URINE VOLUMES ADEQUATE

DROP IN BLOOD PRESSURE

NO THIRST

Fig. 2. Graphic Presentation of Experiment ib. Subject E. B. Dehydration by Means of Constant Jejunal Suction With Miller-Abbott Tube; Water Intake Adequate 
TABLE III

Data on Experiment IIA. Subject E. B. Dehydration by means of water deprivation; no abnormal loss of salt

\begin{tabular}{|c|c|c|c|c|c|c|c|c|c|c|c|c|}
\hline \multirow{2}{*}{ Days } & \multirow{2}{*}{$\begin{array}{l}\text { Body } \\
\text { weight }\end{array}$} & \multirow{2}{*}{$\begin{array}{c}\text { Fluid } \\
\text { intake } \\
\text { (oral) }\end{array}$} & \multicolumn{3}{|c|}{ Urine } & \multicolumn{6}{|c|}{ Blood } & \multirow{2}{*}{ Remarks } \\
\hline & & & Amount & $\mathrm{Na}$ & $\mathbf{C l}$ & $\underset{\text { tocrit }}{\text { Hema- }}$ & $\begin{array}{l}\text { Plasma } \\
\text { protein }\end{array}$ & $\underset{\mathbf{N a}}{\text { Serum }}$ & $\underset{\mathrm{Cl}}{\text { Plagma }}$ & $\begin{array}{r}\text { Plas } \\
\mathrm{CO}_{2} \mathrm{C} . \mathrm{P}\end{array}$ & ma & \\
\hline 0 & $\begin{array}{l}\text { kgm. } \\
57.0\end{array}$ & cc. & $c c$. & grams & grams & $\begin{array}{c}\text { per } \\
\text { cent } \\
39.0\end{array}$ & $\begin{array}{c}\text { grams } \\
\text { per } \\
\text { cent } \\
6.0\end{array}$ & $\begin{array}{c}\underset{\text { per }}{\operatorname{mgm} .} \\
\text { cent } \\
307\end{array}$ & $\begin{array}{c}\text { mgm. } \\
\text { per cent } \\
(\mathrm{as} \mathrm{NaCl}) \\
554\end{array}$ & $\begin{array}{c}\text { oolume } \\
\text { per } \\
\text { cent } \\
57\end{array}$ & $\begin{array}{c}\operatorname{mgm} \\
\text { per } \\
\text { cent }\end{array}$ & \\
\hline $\begin{array}{l}1 \\
2\end{array}$ & $\begin{array}{l}56.9 \\
57.3\end{array}$ & $\begin{array}{l}\text { ad lib. } \\
\text { ad lib. }\end{array}$ & $\begin{array}{l}1310 \\
1360\end{array}$ & $\begin{array}{l}0.14 \\
0.45\end{array}$ & $\begin{array}{l}0.35 \\
0.54\end{array}$ & 40.0 & 6.4 & 299 & 553 & 55 & 33 & $\begin{array}{l}\text { Salt-poor diet. Water ad } \\
\text { libitum. }\end{array}$ \\
\hline $\begin{array}{l}3 \\
4 \\
5 \\
6 \\
7\end{array}$ & $\begin{array}{l}\mathbf{5 6 . 5} \\
\mathbf{5 6 . 0} \\
\mathbf{5 5 . 7} \\
\mathbf{5 4 . 7} \\
\mathbf{5 4 . 9}\end{array}$ & $\begin{array}{r}0 \\
0 \\
0 \\
0 \\
600\end{array}$ & $\begin{array}{c}770 \\
680 \\
1020^{*} \\
600\end{array}$ & $\begin{array}{l}0.88 \\
1.06 \\
\\
1.30^{*} \\
0.58\end{array}$ & $\begin{array}{l}0.49 \\
1.36 \\
\\
2.51^{*} \\
0.87\end{array}$ & $\begin{array}{l}40.3 \\
41.7 \\
41.3\end{array}$ & $\begin{array}{l}6.6 \\
\\
7.4 \\
7.1\end{array}$ & $\begin{array}{l}301 \\
298 \\
292\end{array}$ & $\begin{array}{l}571 \\
\\
584 \\
584\end{array}$ & $\begin{array}{l}57 \\
51 \\
52\end{array}$ & $\begin{array}{l}36 \\
39 \\
40 \\
48\end{array}$ & $\begin{array}{l}\text { Salt-poor dry diet. No fluid } \\
\text { intake for } 4 \text { days. }\end{array}$ \\
\hline 9 & $\begin{array}{l}57.1 \\
57.4\end{array}$ & $\begin{array}{c}3000 \\
+ \text { meals } \\
2000 \\
+ \text { meals }\end{array}$ & $\begin{array}{l}1850 \\
3180\end{array}$ & 0.28 & 0.41 & 41.7 & 6.9 & 279 & 556 & 54 & 26 & Fluids resumed. \\
\hline
\end{tabular}

* Represents 48-hour period, because of failure to collect urine specimen at proper time.

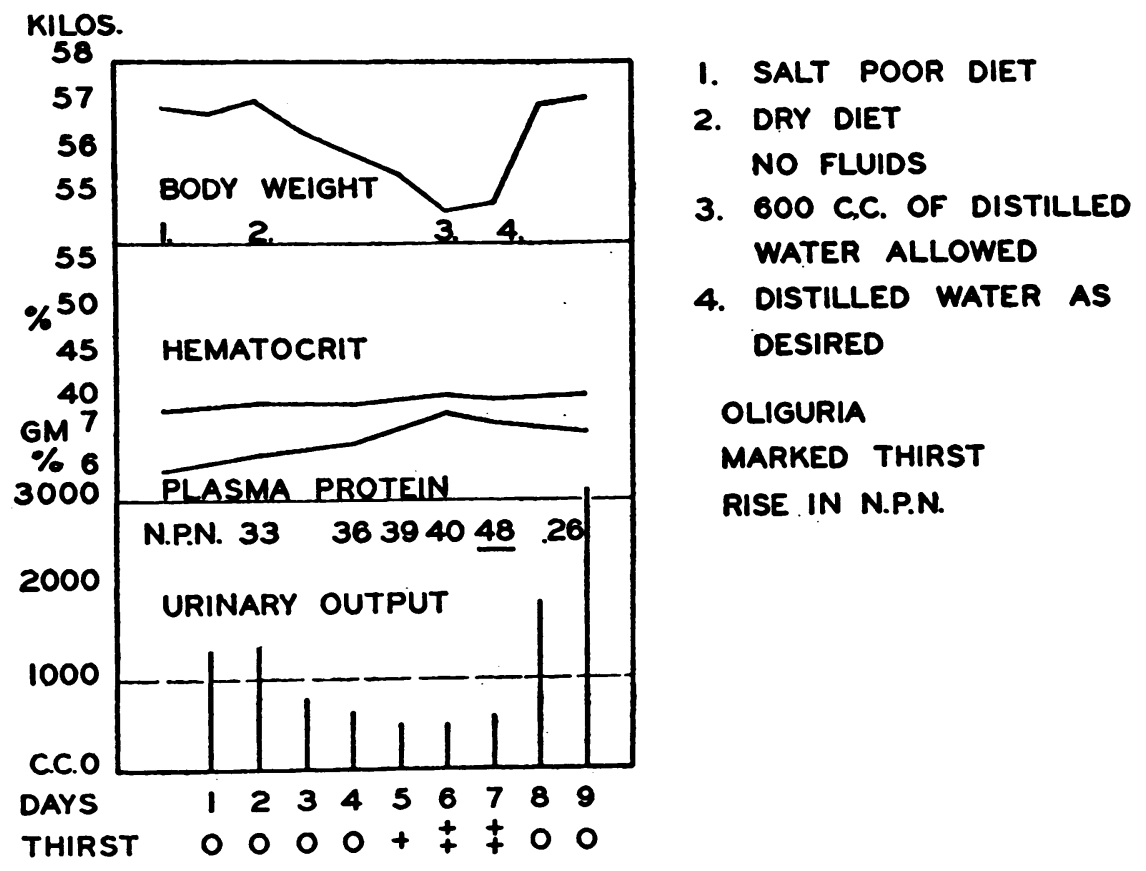

Fig. 3. Graphic Presentation of Experiment ila. Subject E. B. Dehydration by Means of Water Deprivation; No Abnormal Loss of Salt

dration period $600 \mathrm{cc}$. of distilled water were allowed so that the subject could eat his food, yet on this day the urinary output was only 600 cc. and the blood non-protein nitrogen continued to rise.

As soon as the subject was allowed distilled water ad libitum, recovery was prompt. The body weight rose to its previous level, the urine volume increased and the blood non-protein nitrogen returned to normal. The thirst disappeared promptly.

In the last experiment, IIB, dehydration re- 
sulted from deprivation of both food and water and the results were quite similar to those in Experiment IIA. The data in Table IV and Figure 4 show that there occurred a loss of weight, oliguria, azotemia, marked thirst, and very little change in composition of the blood. There was no significant alteration in hematocrit or in plasma protein concentration. As in the previous experiment, the administration of distilled water promptly alleviated the thirst and was followed by a diuresis and a return to normal of the blood non-protein nitrogen. On the first day of the recovery period, the subject was given 4318 grams of water. The first half of this was taken eagerly within about an hour, but the second half was drunk reluctantly and in small amounts throughout the day. The urine volume for that day was $2090 \mathrm{cc}$. and the gain in weight was 1257 grams. On the following day 4000 grams of water were offered, but only 3515 grams were drunk, the subject having to force down most of this. The urinary output for that day was $3110 \mathrm{cc}$. and there was a loss of 449 grams of body weight.

On the 10th day potassium salts were given and produced no significant changes. The subject continued to lose weight until the final day of the experiment, when 9 grams of $\mathrm{NaCl}$ were administered, which resulted in a weight gain of 1070 grams.

In Table IV are shown also other data of interest, such as the specific gravity of the urine and the urinary excretion of nitrogen, sodium, potassium, chloride, and total solids. The specific gravity was high during the 3 days of the dehydration period, being above 1.036 as compared to 1.020 or less on all other days. The excretion of nitrogen averaged about 13 grams during the latter part of the fast and the high value of 16.7 grams occurred with the diuresis on the first day of water administration, the day on which the blood non-protein nitrogen returned to normal. The sodium excretion diminished dur-

TABLE IV

Data on Experiment IIB. Subject T. G. Dehydration by means of total fasting; no abnormal loss of salt

\begin{tabular}{|c|c|c|c|c|c|c|c|c|c|c|c|c|c|c|c|c|c|c|c|c|c|c|}
\hline \multirow[b]{2}{*}{ 空 } & \multirow[b]{2}{*}{ 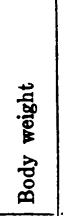 } & \multirow[b]{2}{*}{ 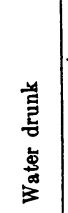 } & \multicolumn{2}{|c|}{ Diet } & \multicolumn{9}{|c|}{ Urine } & \multicolumn{8}{|c|}{ Blood } & \multirow[b]{2}{*}{ Remarks } \\
\hline & & & 䔞 & 莣 & 章 & $\begin{array}{l}\frac{\vec{z}}{\mathrm{c}} \\
\frac{\mathrm{a}}{\mathrm{d}} \\
\mathrm{B}\end{array}$ & 䎹 & 였 & 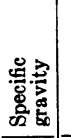 & $z$ & ż & 4 & $\bar{\tau}$ & 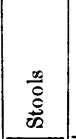 & 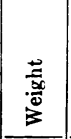 & 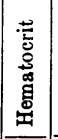 & 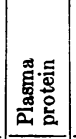 & 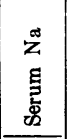 & 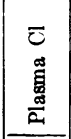 & 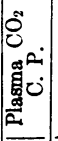 & ż & \\
\hline 0 & $\begin{array}{l}\text { grams } \\
74596\end{array}$ & grams & & grams & $c c$. & grams & grams & grams & & grams & grams & grams & grams & grams & $\begin{array}{c}\text { grams } \\
40 \\
\end{array}$ & $\begin{array}{c}\text { per } \\
\text { cent } \\
47.8 \\
\end{array}$ & $\begin{array}{c}\text { grams } \\
\text { per } \\
\text { cent } \\
7.7\end{array}$ & $\begin{array}{c}\text { mom. } \\
\text { per } \\
\text { cent } \\
292 \\
\end{array}$ & $\begin{array}{c}\text { mom. } \\
\text { per } \\
\text { cent } \\
578\end{array}$ & $\begin{array}{l}\text { ool } \\
\text { ume } \\
\text { per } \\
\text { cent } \\
48 \\
\end{array}$ & $\begin{array}{c}\text { mom. } \\
\text { per } \\
\text { cent }\end{array}$ & \\
\hline 1 & 73949 & $\begin{array}{l}\text { ad } \\
\text { lib. }\end{array}$ & $\begin{array}{l}\text { salt } \\
\text { poor }\end{array}$ & $\begin{array}{l}\text { ad } \\
\text { lib. }\end{array}$ & 1520 & $\overline{1549}$ & $\overline{47.8}$ & $\overline{1501}$ & $\overline{1.019}$ & 13.8 & 2.23 & 2.78 & 3.48 & & & & & & & & & \\
\hline 2 & 74031 & $\begin{array}{l}\text { ad } \\
\text { lib. }\end{array}$ & $\begin{array}{l}\text { salt } \\
\text { poor }\end{array}$ & $\begin{array}{l}\text { ad } \\
\text { lib. }\end{array}$ & 1535 & 1566 & 58.6 & 1507 & 1.020 & 17.0 & 0.84 & 2.53 & 1.53 & & 30 & & 7.4 & 279 & 576 & 46 & & $\begin{array}{l}\text { Preliminary period. } \\
\text { Salt-poor diet. }\end{array}$ \\
\hline 3 & 74091 & $\begin{array}{l}\text { ad } \\
\text { lib. }\end{array}$ & $\begin{array}{l}\text { salt } \\
\text { poor }\end{array}$ & $\begin{array}{l}\text { ad } \\
\text { lib. }\end{array}$ & 1120 & 1148 & 61.0 & 1077 & 1.020 & 17.5 & 0.46 & 2.90 & 1.00 & & 5 & 48.6 & 7.9 & & & & & \\
\hline 4 & 73901 & $\begin{array}{l}\text { ad } \\
\text { lib. }\end{array}$ & $\begin{array}{l}\text { sslt } \\
\text { poor }\end{array}$ & $\begin{array}{l}\text { ad } \\
\text { lib. }\end{array}$ & 1330 & 1355 & 58.0 & 1297 & 1.019 & 16.8 & 0.24 & 2.58 & 0.76 & & 45 & 48.4 & 7.5 & 301 & 576 & 48 & 39 & \\
\hline $\begin{array}{l}5 \\
6 \\
7 \\
\end{array}$ & \begin{tabular}{|l|}
72504 \\
71106 \\
69777 \\
\end{tabular} & $\begin{array}{l}0 \\
0 \\
0 \\
\end{array}$ & $\begin{array}{l}0 \\
0 \\
0 \\
\end{array}$ & $\begin{array}{l}0 \\
0 \\
0 \\
\end{array}$ & $\begin{array}{l}430 \\
450 \\
445 \\
\end{array}$ & $\begin{array}{l}445 \\
467 \\
462 \\
\end{array}$ & \begin{tabular}{|l|}
36.2 \\
39.5 \\
37.6 \\
\end{tabular} & $\begin{array}{l}409 \\
428 \\
424 \\
\end{array}$ & \begin{tabular}{|l|}
1.036 \\
1.037 \\
1.038 \\
\end{tabular} & $\begin{array}{l}10.2 \\
11.3 \\
11.2 \\
\end{array}$ & \begin{tabular}{l|}
0.13 \\
0.34 \\
0.48 \\
\end{tabular} & \begin{tabular}{|l|}
2.10 \\
2.02 \\
1.74 \\
\end{tabular} & \begin{tabular}{|l|}
0.65 \\
0.79 \\
0.67 \\
\end{tabular} & $\begin{array}{l}0 \\
0 \\
0 \\
\end{array}$ & $\begin{array}{l}40 \\
40 \\
40 \\
\end{array}$ & \begin{tabular}{|l|}
49.7 \\
51.6 \\
49.2 \\
\end{tabular} & $\begin{array}{l}8.0 \\
8.1 \\
7.7 \\
\end{array}$ & \begin{tabular}{l|}
301 \\
298 \\
309
\end{tabular} & $\begin{array}{l}574 \\
584 \\
597\end{array}$ & $\begin{array}{l}48 \\
49 \\
31\end{array}$ & $\begin{array}{l}40 \\
42 \\
49\end{array}$ & $\begin{array}{l}\text { Total fast. } \\
\text { No food or water. }\end{array}$ \\
\hline $\begin{array}{l}8 \\
9 \\
\end{array}$ & 71034 & \begin{tabular}{|l}
4318 \\
3510 \\
\end{tabular} & $\begin{array}{l}0 \\
0\end{array}$ & $\begin{array}{l}0 \\
0\end{array}$ & $\begin{array}{l}2090 \\
3110\end{array}$ & $\begin{array}{l}2117 \\
3132 \\
\end{array}$ & $\begin{array}{l}44.5 \\
30.2 \\
\end{array}$ & $\begin{array}{l}2072 \\
3102 \\
\end{array}$ & \begin{tabular}{|l|}
1.013 \\
1.007 \\
\end{tabular} & $\begin{array}{l}16.7 \\
12.9\end{array}$ & $\begin{array}{l}0.33 \\
0.21\end{array}$ & $\begin{array}{l}1.62 \\
1.80 \\
\end{array}$ & $\begin{array}{l}0.32 \\
0.30 \\
\end{array}$ & $\begin{array}{l}0 \\
0\end{array}$ & $\begin{array}{l}40 \\
35 \\
\end{array}$ & \begin{tabular}{|l|}
50.3 \\
49.0
\end{tabular} & $\begin{array}{l}7.7 \\
7.7 \\
\end{array}$ & $\begin{array}{l}300 \\
303 \\
\end{array}$ & $\begin{array}{l}561 \\
556 \\
\end{array}$ & $\begin{array}{l}47 \\
43 \\
\end{array}$ & 31 & $\begin{array}{l}\text { Distilled water ad } \\
\text { libitum. }\end{array}$ \\
\hline$\overline{10}$ & $\overline{70325}$ & $\overline{3298^{*}}$ & 0 & 0 & 2500 & $\overline{2527}$ & $\overline{46.8}$ & $\overline{2480}$ & $\overline{1.011}$ & $\overline{13.2}$ & $\overline{0.36}$ & $\overline{3.06}$ & 0.33 & 175 & 30 & $\overline{50.1}$ & 8.1 & 289 & 545 & $\overline{40}$ & & $\begin{array}{l}\text { Potassium salts } \\
\text { given. }\end{array}$ \\
\hline$\overline{11}$ & $\overrightarrow{70042}$ & $\overline{2143}$ & $\begin{array}{l}\text { whole } \\
\text { milk }\end{array}$ & $\overline{2074}$ & 2550 & 3578 & $\overline{43.0}$ & $\overline{3535}$ & $\overline{1.008}$ & $\overline{15.3}$ & $\overline{0.38}$ & $\overline{1.76}$ & $\overline{0.39}$ & 0 & 30 & $\overline{47.7}$ & 7.5 & 303 & 563 & 45 & & $\overline{\text { Milk } 2074 \text { grams. }}$ \\
\hline$\overline{12}$ & $\overline{71112}$ & $2687 \dagger$ & $\begin{array}{l}\text { whole } \\
\text { milk }\end{array}$ & $\overline{2083}$ & $\overline{1880}$ & $\overline{1899}$ & $\overline{35.0}$ & $\overline{1864}$ & $\overline{1.010}$ & $\overline{10.4}$ & 0.26 & 2.11 & $\overline{0.72}$ & 0 & 30 & $\overline{45.1}$ & 7.4 & & 576 & 45 & & $\begin{array}{l}\text { Milk } 2083 \text { grams. } \\
\mathrm{NaCl} 9.0 \text { grams. }\end{array}$ \\
\hline
\end{tabular}

* Given with 4.8 grams of $\mathrm{K}$ as a mixture of $\mathrm{K}_{2} \mathrm{HPO}_{4}$ and $\mathrm{KH}_{2} \mathrm{PO}_{4}$.

$\dagger$ Given with 9 grams of $\mathrm{NaCl}$. 


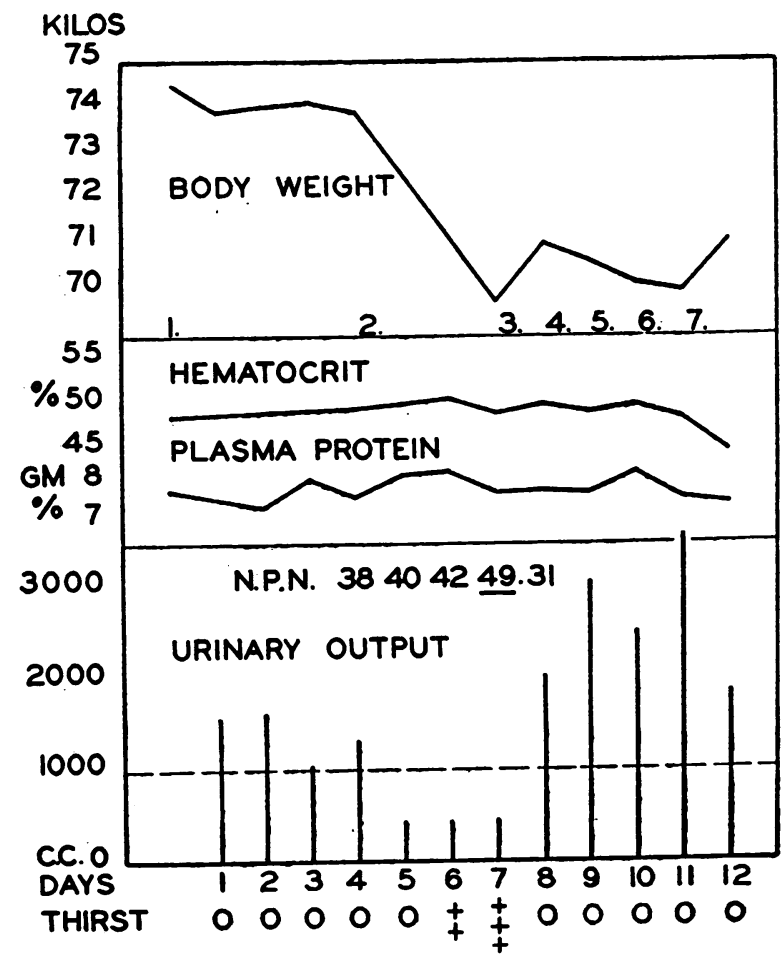

I. SALT POOR DIET

2. TOTAL FAST

(NO FOOD OR WATER)

3. WATER (DISTILLED)

4318 C.C.

4. WATER 3515 C.C.

5. POT.PHOSPHATE (4.8.GM. OF K)

I WATER 3298 C.C.

6. WHOLE MILK 2000 C.C.

\& WATER 2143 C.C.

7. NACL $9.0 \mathrm{GM}$.

MILK 2000 C.C.

WATER 1687 C.C

OLIGURIA

MARKED THIRST

RISE IN N.P.N.

Fig. 4. Graphic Presentation of Experiment ilb. Subject T. G. Dehydration by Means of Total Fasting; No Abnormal Loss of Salt

ing the preliminary period with the salt-poor diet and remained at a low level throughout, though there was a slight rise at the time of maximum dehydration. The chloride excretion paralleled in a general way the sodium excretion. During the fast, potassium excretion remained close to the average of 1.9 grams per day except on the day the potassium salts were administered, when it rose to 3.06 grams. The excretion of urinary solids averaged 39.1 grams per day during the fasting period.

Calculations of water and energy balance in Experiment IIB were made according to methods recommended by Newburgh (11), and are summarized in Table $V$. The values for utilization of protein are derived from the values for nitrogen excretion, a suitable correction being made for the days of water deprivation while nitrogen was being retained and for the day immediately afterward, when the retained nitrogen was being washed out. The values for carbohydrate utilization during the initial days of fasting are estimates based upon other studies of fasting human beings $(12,13)$. During the last 2 days of the experiment it was assumed that no glycogen was stored inasmuch as the diet was submaintenance.

The values for water balance are shown in the last column of Table V. There was a negative balance of 2088 grams during the period of dehydration, and a positive balance of 1800 grams on the first day of recovery, when water was allowed ad libitum. The only other large change in water balance was on the final day, when 9 grams of $\mathrm{NaCl}$ were ingested, which resulted in a positive balance of 1064 grams.

In order to compare the water balance with the balances of sodium and potassium, some additional data are presented in Table VI. This table includes the 3 days of water deprivation, together with the 2 following days, during which nothing but distilled water was administered. During these 5 days all balances, except for water, were necessarily negative. In columns 2 and 3 are given the balances for $\mathrm{Na}$ and $\mathrm{K}$ respectively, expressed in milliequivalents. The figures in column 4 refer to the amount of extracellular water which would ordinarily correspond to the sodium balance for the period in question. The figures 
TABLE V

Further data from Experiment IIB. Subject T. G. Energy and water balance during total fast and during recovery period

\begin{tabular}{|c|c|c|c|c|c|c|c|c|c|c|c|c|c|c|c|c|c|}
\hline \multirow{2}{*}{ Days } & \multirow{2}{*}{$\begin{array}{c}\text { Change } \\
\text { in body } \\
\text { weight }\end{array}$} & \multirow{2}{*}{ I. L. } & \multicolumn{3}{|c|}{ Metabolic mixture } & \multirow{2}{*}{$\begin{array}{l}\text { Cal- } \\
\text { ories }\end{array}$} & \multicolumn{10}{|c|}{ Water } & \multirow[b]{2}{*}{ Remarks } \\
\hline & & & $\mathrm{CHO}$ & $\underset{(\operatorname{as} \mathbf{N})}{\mathbf{P}}$ & Fat & & $\underset{\text { drunk }}{\mathrm{H}_{2} \mathrm{O}}$ & $\begin{array}{c}\mathrm{H}_{2} \mathrm{O} \\
\text { of } \\
\text { food }\end{array}$ & $\begin{array}{l}\text { Oxi- } \\
\text { da- } \\
\text { tion }\end{array}$ & $\begin{array}{c}\text { Pre- } \\
\text { formed }\end{array}$ & I. W. & Urine & Blood & $\begin{array}{c}\text { Total } \\
\text { in }\end{array}$ & $\begin{array}{c}\text { Total } \\
\text { out }\end{array}$ & Balance & \\
\hline \multirow[t]{2}{*}{$\begin{array}{l}5 \\
6 \\
7\end{array}$} & \multirow[t]{2}{*}{$\begin{array}{l}\text { grams } \\
-1397 \\
-1398 \\
-1329\end{array}$} & \multirow[t]{2}{*}{$\begin{array}{c}\text { grams } \\
912 \\
891 \\
827\end{array}$} & \multirow[t]{2}{*}{$\begin{array}{c}\text { grams } \\
100 \\
50 \\
0\end{array}$} & \multirow[t]{2}{*}{$\begin{array}{l}\text { grams } \\
11.0 \\
12.4 \\
13.0\end{array}$} & \multirow[t]{2}{*}{$\begin{array}{c}\text { grams } \\
142 \\
160 \\
170\end{array}$} & \multirow[t]{2}{*}{$\begin{array}{l}2025 \\
2022 \\
1926\end{array}$} & \multirow[t]{2}{*}{\begin{tabular}{|c|} 
grams \\
$\mathbf{0}$ \\
$\mathbf{0}$ \\
$\mathbf{0}$
\end{tabular}} & \multirow[t]{2}{*}{$\begin{array}{r}\text { grams } \\
\mathbf{0} \\
\mathbf{0} \\
\mathbf{0}\end{array}$} & \multirow[t]{2}{*}{$\begin{array}{c}\text { grams } \\
241 \\
234 \\
216\end{array}$} & \multirow[t]{2}{*}{\begin{tabular}{|r|} 
grams \\
520 \\
395 \\
261
\end{tabular}} & \multirow[t]{2}{*}{$\begin{array}{c}\text { grams } \\
873 \\
871 \\
830\end{array}$} & \multirow[t]{2}{*}{$\begin{array}{c}\text { grams } \\
409 \\
428 \\
424\end{array}$} & \multirow[t]{2}{*}{$\begin{array}{c}\text { grams } \\
40 \\
40 \\
40\end{array}$} & \multirow[t]{2}{*}{$\begin{array}{c}\text { grams } \\
761 \\
629 \\
477\end{array}$} & \multirow[t]{2}{*}{$\begin{array}{l}\text { grams } \\
1322 \\
1339 \\
1294\end{array}$} & $\begin{array}{c}\text { grams } \\
-561 \\
-710 \\
-817\end{array}$ & \multirow[t]{2}{*}{ Total fast. } \\
\hline & & & & & & & & & & & & & & & & -2088 & \\
\hline \multirow[t]{2}{*}{$\begin{array}{l}8 \\
9\end{array}$} & \multirow[t]{2}{*}{$\begin{array}{l}+1257 \\
-449\end{array}$} & \multirow[t]{2}{*}{$\begin{array}{l}904 \\
797\end{array}$} & \multirow[t]{2}{*}{$\begin{array}{l}\mathbf{0} \\
\mathbf{0}\end{array}$} & \multirow[t]{2}{*}{$\begin{array}{l}13.0 \\
12.9\end{array}$} & \multirow[t]{2}{*}{$\begin{array}{l}189 \\
163\end{array}$} & \multirow[t]{2}{*}{$\begin{array}{l}2107 \\
1853\end{array}$} & \multirow[t]{2}{*}{$\begin{array}{l}4318 \\
3515\end{array}$} & \multirow[t]{2}{*}{$\begin{array}{l}\mathbf{0} \\
\mathbf{0}\end{array}$} & \multirow[t]{2}{*}{$\begin{array}{l}236 \\
207\end{array}$} & \multirow[t]{2}{*}{$\begin{array}{l}262 \\
258\end{array}$} & $\begin{array}{l}904 \\
799\end{array}$ & $\begin{array}{l}2072 \\
3102\end{array}$ & $\begin{array}{l}40 \\
35\end{array}$ & $\begin{array}{l}4816 \\
3980\end{array}$ & $\begin{array}{l}3016 \\
3936\end{array}$ & $\begin{array}{r}+1800 \\
+\quad 44 \\
\end{array}$ & $\begin{array}{c}\text { Distilled } \mathrm{H}_{2} \mathrm{O} \\
\text { ad libitum. }\end{array}$ \\
\hline & & & & & & & & & & & & & & & & +1844 & \\
\hline 10 & -260 & 826 & 0 & 13.2 & 169 & 1922 & 3298 & 0 & 215 & 264 & 828 & 2480 & 30 & 3777 & 3496 & +281 & $\begin{array}{l}\text { Potassium salts } \\
\text { administered. }\end{array}$ \\
\hline $\begin{array}{l}11 \\
12\end{array}$ & $\begin{array}{r}289 \\
+1070\end{array}$ & $\begin{array}{l}892 \\
780\end{array}$ & $\begin{array}{l}104 \\
104\end{array}$ & $\begin{array}{l}15.3 \\
10.4\end{array}$ & $\begin{array}{l}121 \\
108\end{array}$ & $\begin{array}{l}1958 \\
1710\end{array}$ & $\begin{array}{l}2143 \\
1687\end{array}$ & $\begin{array}{l}1804 \\
1812\end{array}$ & $\begin{array}{l}232 \\
205\end{array}$ & $\begin{array}{r}86 \\
-9\end{array}$ & $\begin{array}{l}846 \\
737\end{array}$ & $\begin{array}{l}3535 \\
1864\end{array}$ & $\begin{array}{l}30 \\
30\end{array}$ & $\begin{array}{l}4265 \\
3695\end{array}$ & $\begin{array}{l}4411 \\
2631\end{array}$ & $\begin{array}{r}146 \\
+1064 \\
\end{array}$ & $\begin{array}{lll}\text { Milk } 2074 & \text { grams. } \\
\text { Milk } 2083 \text { grams. }\end{array}$ \\
\hline & & & & & & & & & & & & & & & & +918 & \\
\hline Total & -2495 & & & & & & & & & 2037 & & & 285 & 22400 & 21445 & +955 & \\
\hline
\end{tabular}

TABLE VI

Further data from Experiment IIB. Subject T. G. Relationship of water balance to balance of Na and $K$ during the fasting period

\begin{tabular}{|c|c|c|c|c|c|c|c|c|c|}
\hline$\underset{1}{\text { Column }}$ & 2 & 3 & 4 & 5 & 6 & 7 & 8 & 9 & \\
\hline Days & $\begin{array}{c}\mathrm{Na} \\
\text { balance }\end{array}$ & $\underset{\text { balance }}{\mathrm{K}}$ & 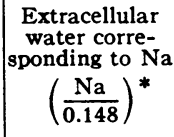 & $\begin{array}{c}\text { Intracellular } \\
\text { water corre- } \\
\text { sponding to } \mathrm{K} \\
\left(\frac{\mathrm{K}-0.02 \mathrm{Na}}{0.14}\right)^{*}\end{array}$ & $\begin{array}{c}\text { "Pre-, } \\
\text { formed" } \\
\text { water }\end{array}$ & $\begin{array}{l}\text { Balance } \\
\text { of "avail- } \\
\text { able" } \\
\text { water }\end{array}$ & $\begin{array}{c}\text { Absolute } \\
\text { water bal- } \\
\text { ance (Column } \\
\text { minus } \\
\text { Column 6) }\end{array}$ & $\begin{array}{c}\text { Water losses not } \\
\text { accounted for by } \\
\text { losses of Na and } \mathrm{K} \\
\text { (Column } 8 \text { minus } \\
\text { Columns } 4 \text { and } 5 \text { ) }\end{array}$ & Remarks \\
\hline \multirow[t]{2}{*}{$\begin{array}{l}5 \\
6 \\
7\end{array}$} & $\begin{array}{l}m . e q . \\
-5.6 \\
-14.6 \\
-21.0\end{array}$ & $\begin{array}{r}m . e q . \\
-53.8 \\
=51.6 \\
-\quad 44.7\end{array}$ & $\begin{array}{l}\text { grams } \\
-\quad 38 \\
-98 \\
-142\end{array}$ & $\begin{array}{l}\text { grams } \\
=384 \\
=367 \\
-316\end{array}$ & $\begin{array}{r}\text { grams } \\
520 \\
395 \\
261\end{array}$ & $\begin{array}{l}\text { grams } \\
-561 \\
=710 \\
-817\end{array}$ & $\begin{array}{l}\text { grams } \\
-1081 \\
-1105 \\
-1078\end{array}$ & $\begin{array}{l}\text { grams } \\
=659 \\
=640 \\
-620\end{array}$ & \multirow[t]{2}{*}{ Total fast. } \\
\hline & -41.2 & -150.2 & -278 & -1067 & 1176 & -2088 & -3264 & -1919 & \\
\hline \multirow[t]{2}{*}{$\begin{array}{l}8 \\
9\end{array}$} & $\begin{array}{r}-14.4 \\
-9.0\end{array}$ & $\begin{array}{l}-41.4 \\
-46.1\end{array}$ & $\begin{array}{l}-97 \\
-61\end{array}$ & $\begin{array}{l}-294 \\
-328\end{array}$ & $\begin{array}{l}262 \\
258\end{array}$ & $\begin{array}{r}+1800 \\
+\quad 44\end{array}$ & $\begin{array}{r}+1538 \\
-\quad 214\end{array}$ & $\begin{array}{r}1929 \\
+\quad 175\end{array}$ & \multirow{2}{*}{$\begin{array}{l}\text { Distilled water by } \\
\text { mouth ad libitum. } \\
\text { No diet. }\end{array}$} \\
\hline & -23.4 & -87.5 & -158 & -622 & 520 & +1844 & +1324 & +1754 & \\
\hline Total & -64.6 & -237.7 & -436 & -1689 & 1696 & -244 & -1940 & -165 & \\
\hline
\end{tabular}

* See text for explanation of formulae.

in column 5 refer to the amount of intracellular water which would ordinarily correspond to the potassium balance for the period in question, a correction being made in each case for the potassium present in extracellular fluid. These figures in columns 4 and 5 are derived from those in columns 2 and 3 by applying the formulae indicated in the table. The formulae are those recommended by Peters (14), except that they have been modified to conform with more recent analyses of extracellular fluid as suggested by Gamble
(15). It is obvious that in this type of experiment the values in columns 4 and 5 cannot possibly express actual changes in water balance of intracellular or extracellular water for the simple reason that these values during the 5 days covered by the table are all necessarily negative, even on the day when the water balance was positive. The values for "preformed water" have been carried over from the preceding table and placed in column 6. The figures in column 7 are carried over from the last column of Table V. Columns 
8 and 9 are self-explanatory and will be discussed later.

\section{DISCUSSION}

\section{The two types of dehydration}

The results of these experiments in human beings substantiate the findings of Kerpel-Fronius in rabbits (1). It appears that the two types of dehydration in question differ from each other not only in mechanism of production but also in symptomatology and in the treatment indicated. In fact, about the only similarity between the two conditions is that implied by the term "dehydration."

The body is continually losing water insensibly by evaporation, frequently more than 1000 or 1200 grams daily. This insensible water ordinarily contains a negligible amount of salt when sweating is avoided (16) and can, therefore, be considered essentially a loss of distilled water. If this loss plus the losses in urine and feces are not replaced by the water of food and drink plus the water available from other sources (water of oxidation and "preformed water"), a condition of true dehydration results, with thirst, oliguria, and a rising blood non-protein nitrogen. There occurs a water shortage in all parts of the body, intracellular as well as extracellular. For if such dehydration were assumed to be localized, let us say, to the extracellular portion of the body, we would then have a hypertonicity of the extracellular fluid as compared to intracellular fluid. The laws of membrane equilibrium demand that the diffusible particles shift until osmotic equilibrium is reached. But since the cell wall is not freely permeable to the most important ions, $\mathrm{Na}^{+}$and $\mathrm{K}^{+}$, this can only mean that water must pass out of the cells until equilibrium is reached. Thus, in water deprivation we are dealing with a diffuse, and not a localized dehydration. This concept fits in well with the fact that only slight changes were observed in the hematocrit during this type of dehydration (Figures 3 and 4 ).

In the salt-loss type of dehydration, on the other hand, we have a radically different state of affairs. As long as the water intake is adequate, no thirst occurs. There is no shortage of water as such. The deficiency of sodium, however, results in a reduction in the volume of the extra- cellular fluid $(2,17,18)$, which can be thought of as a localized type of dehydration. If we were to postulate that a large loss of sodium could occur without a resulting loss of extracellular water, we should then be postulating a hypotonicity of the extracellular fluid as compared to the intracellular fluid. Such a system would not be in osmotic equilibrium and, therefore, could not endure. Water would be forced to leave the extracellular spaces and either leave the body or pass into the cells, or both, until equilibrium were reached. The net result, therefore, would be a localized dehydration involving the extracellular fluid. Experimental evidence that this actually occurs has been presented by Darrow and Yannet (18). In Experiments IA and IB there was no thirst, and attempts to replace the depleted extracellular fluid with salt-free water merely resulted in a corresponding increase in urinary output. The manifestations of extracellular desiccation are those of peripheral circulatory failure. The reduced extracellular volume includes a reduced plasma volume as indicated by a rise in the hematocrit (Figures 1 and 2). Such a reduction in blood volume results in apathy, weakness, fainting, anorexia, low blood pressure and, if the condition is allowed to progress far enough, to circulatory collapse. This "electrolyte shock," as we have termed it, may be indistinguishable clinically from shock due to other causes, and is brought to mind by the realization that the patient has been losing appreciable amounts of electrolyte-containing fluid. If the blood pressure does not fall too low, and if the water intake is adequate, the urinary volume may remain normal, as was the case in Experiments IA and IB.

Our results and interpretation of the experiments, here described, fit in very well with the demineralization experiments in dogs described by Darrow and Yannet (18). These authors clearly demonstrated the relationship of salt loss to extracellular dehydration. They noted also that "thirst is not an obligatory accompaniment of dehydration" and that "water intake and urine output may be normal in the presence of dehydration."

An interesting and important practical question is the following: In the extracellular type of dehydration, to what extent is the blood plasma 
affected? One often hears the statement that in dehydration the plasma volume is protected by the existence of a large amount of fluid in the interstitial reservoir and that no reduction in plasma volume occurs until dehydration is quite advanced. The results of our experiments do not support this concept. In both of our salt depletion experiments we were impressed by the fact that evidence of hemoconcentration appeared within 24 hours after insertion of the MillerAbbott tube and increased on each succeeding day until the tube was removed. We did not make actual determinations of plasma volumes in our subjects. However, if one considers the blood volumes of W. M. and E. B. to be 5250 and 4500 cc., respectively (computed as 7.5 per cent of body weight), then, since the initial hematocrits were 47.2 and 44.3 per cent, the estimated plasma volumes would be $2772 \mathrm{cc}$. for W. M. and $2506 \mathrm{cc}$. for $\mathrm{E}$. B. If we use the change in hematocrit to indicate the change in plasma volume, we arrive at a reduction of $1101 \mathrm{cc}$. (or 39 per cent) for W. M. and $1008 \mathrm{cc}$. (or 40 per cent) for E. B. This would correspond to reductions in blood volume of 21 and 22 per cent, respectively, reductions which are of sufficient magnitude to produce shock (19). These calculations give minimum values, as they are based upon the assumption that the total cell volumes remained constant, which was not the case since a considerable amount of blood was taken for the various blood tests (perhaps 200 to $300 \mathrm{cc}$. during the period in question).

It seems reasonable to conclude, therefore, that serious depletion of extracellular electrolyte causes a decrease in the volume of extracellular fluid, both interstitial fluid and plasma. This leads eventually to peripheral circulatory failure, but before this stage is reached a progressive hemoconcentration can be observed. Thus, frequent hematocrit and plasma protein determinations are of definite value in connection with the diagnosis and treatment of this type of dehydration, a fact which has been emphasized recently by Scudder (20) and by others $(21,22)$.

\section{Mixed type of dehydration}

It is very common to encounter patients with both the "salt-loss" and the "water deprivation" type of dehydration existing simultaneously. One of the most frequent causes of dehydration is vomiting, and this generally gives rise to the mixed type. However, this need not always be the case. If vomiting is slight and anorexia marked, it may lead to severe water deprivation without significant salt loss. On the other hand, if vomiting is marked and at the same time is characterized by remissions during which the patient can drink, there may develop a severe electrolyte loss without water deprivation and without thirst. As a rule, vomiting by itself does not result in circulatory collapse, though it does predispose to this condition. Since the ratio of sodium to chloride is low in vomitus, severe depletion of sodium is not likely to occur early and therefore the presenting symptoms of a vomiting patient are more likely to be those associated with thirst and occasionally alkalosis.

In diarrheal disease accompanied by vomiting, we also are likely to find a mixed picture, depending upon which factors predominate in any given case. With diarrhea or ileostomy drainage the relative loss of fixed base is greater than in vomiting and, therefore, the likelihood of circulatory collapse is greater.

If a patient with mixed dehydration is treated either by physiological saline alone or by glucose solution alone, the dehydration may then become converted into one or the other of the pure types. We have seen this actually happen.

The fact that dehydration so commonly occurs in mixed form is probably responsible for much of the confusion that exists. Most textbooks do not distinguish between the two types of dehydration. We often encounter seemingly conflicting statements concerning the nature and treatment of dehydration when in reality the statements apply to entirely different conditions.

\section{Dehydration by means of total fasting}

Referring to Table $\mathrm{V}$, it is interesting to note the figures for "preformed water" and how they are related to the water balance the first 3 days of the fasting period. While the glycogen stores in the liver are being used up, the corresponding preformed water becomes available and this tends to retard the dehydration. Definite thirst did not occur until noon of the second day.

Another point of interest is the fact that on 
the first day of recovery this subject gained 1257 grams in weight, in spite of the fact that he was still fasting and losing solids from his body. He was losing solids in two ways. He excreted 44.5 grams in the urine, and oxidized about 229 grams of protein and fat. We should expect, then, that the absolute gain in body water on that day ought to be close to the sum of these three figures, or 1530.5 grams. Table VI gives essentially the same value in column 8 , namely 1538 grams. In other words, here is clear evidence, if any evidence is needed, that a thirsting individual can take up water and retain it without any concomitant intake of electrolytes or of any other solid matter. Wiley and Wiley (23) came to the conclusion that a dehydration of about 1.5 per cent of the body weight can occur without great disturbance of salt balance. Our data indicate that a change in hydration amounting to at least 2 per cent of the body weight can occur without a corresponding change in salt or solid content. In fact, if we correct for the fact that solutes were being lost while water was being gained, as in column 9 in Table VI, we arrive at 1929 grams for the 24-hour period in question, or 2.7 per cent of the body weight. This clearly indicates that for short periods of time water balance cannot always be estimated accurately from balances of $\mathrm{Na}$ and $\mathrm{K}$.

As indicated in column 9 in Table VI, there was, during the 3 days of total fasting, a greater absolute reduction in the amount of body water than would be predicted purely on the basis of sodium and potassium balance. In other words, by the end of the dehydration period there was in the body enough fixed base ( $\mathrm{Na}$ and $\mathrm{K}$ ) to " hold" an additional 1919 grams of water. It is interesting to note that at the end of the following day, during which time water was allowed ad libitum and during which time $\mathrm{Na}$ and $\mathrm{K}$ were still being excreted, the body had regained and retained 1929 grams of water, almost precisely the theoretically predicted amount.

Once the subject was rehydrated, it was not possible to hyperhydrate him by forcing water. The body weight curve seemed to be unaffected by attempts at hyperhydration, although it had been markedly affected by dehydration and rehydration. Of course, the subject was not allowed to drink for several hours immediately preceding each weighing, and was always weighed with an empty bladder.

\section{The signifiance of thirst in dehydration}

As discussed, it is clear that thirst is not present in all types of dehydration. It is present in that type brought about by water deprivation. It may be completely absent in the extracellular, or salt-loss, type of dehydration. These facts fit in well with the concept that thirst depends upon cellular dehydration (24).

\section{Oliguria, anuria, and azotemia}

Oliguria, anuria and azotemia have long been considered common manifestations in dehydrated patients, but the type of dehydration referred to has generally not been specified.

In the experiments described above it is noteworthy that in IIA and IIB, which illustrate the " thirst" type of dehydration, the daily volume of urine decreased to $510 \mathrm{cc}$. in one case and 430 cc. in the other. It will be noted in Experiment IIB (Table IV) that the urine volumes on the 3 days of water deprivation were 430,450 and 445 cc., respectively, the lowest volume occurring on the first day. Obviously, in this case the degree of oliguria is not an accurate index of the extent of the dehydration. In two similar cases reported by Coller and Maddock (25) there was no accurate correlation between the degree of oliguria and the extent of dehydration. It appears that a certain minimum urinary volume is inevitable as long as blood pressure is high enough to insure glomerular filtration. On theoretical grounds we should rarely expect to find anuria from water deprivation alone.

In Experiments IA and IB the urine volumes were normal at all times because water was being adequately supplied. These experiments were terminated before the blood pressures dropped to dangerously low levels. It seems obvious that, if the salt loss had been allowed to continue until true shock occurred, the urine secretion might have ceased altogether for the simple reason that glomerular filtration cannot occur when the blood pressure is sufficiently low (26). It is a common observation that patients in profound shock usually have anuria. 
It seems reasonable to conclude that in the "thirst" type of dehydration associated with simple water deprivation, oliguria should be expected, but not anuria; while in the "salt-loss" type, urinary excretion may vary from the normal to complete anuria.

Azotemia, when due to dehydration, is most simply explained as depending chiefly upon the degree and duration of the associated oliguria or anuria. The extent to which azotemia may depend upon other factors will not be discussed here.

\section{SUMMARY AND CONCLUSIONS}

1. Two distinct types of dehydration were produced in normal human subjects.

2. The results were quite comparable to those previously described in rabbits by Kerpel-Fronius and indicate that the two types differ from each other in mechanism of production, in manifestations, and in the treatment indicated.

3. Dehydration resulting from simple water deprivation is characterized by thirst and oliguria, does not lead to impairment of the circulation, and is completely relieved by the administration of water.

4. Dehydration resulting from abnormal salt loss results mainly in a loss of extracellular fluid, a reduction in plasma volume, and disturbances in the circulation. It is neither characterized by thirst nor relieved by the administration of saltfree fluids, but is promptly relieved by fluid containing sodium chloride.

5. A detailed study was made of the balances of energy, water, sodium, and potassium during a period of total fasting and recovery. The results indicate that during dehydration and recovery the balances of sodium and potassium do not necessarily correspond to the water balance over short periods of time.

The authors wish to acknowledge the helpful advice given by Drs. L. H. Newburgh and C. F. McKhann throughout the course of this investigation.

\section{BIBLIOGRAPHY}

1. Kerpel-Fronius, E., Über die Beziehungen zwischen Salz- und Wasserhaushalt bei experimentellen Wasserverlusten. Ztschr. f. Kinderh., 1935, 57, 489.
2. Idem, Durstexsikkose und Salzmangelexsikkose. Acta Paediat., 1937, 22, 143.

3. Miller, T. G., and Abbott, W. O., Intestinal intubation: a practical technique. Am. J. M. Sc., 1934, 187, 595.

4. Butler, A. M., and Tuthill, E., An application of the uranyl zinc acetate method for determination of sodium in biological material. J. Biol. Chem., 1931, 93, 171.

5. Shohl, A. T., and Bennett, H. B., A micro-method for the determination of potassium as iodoplatinate. J. Biol. Chem., 1928, 78, 643.

6. Wilson, D. W., and Ball, E. G., A study of the estimation of chloride in blood and serum. J. Biol. Chem., 1928, 79, 221.

7. Shackell, L. F., An improved method of desiccation, with some applications to biological problems. Am. J. Physiol., 1909, 24, 325.

8. Van Slyke, D. D., and Cullen, G. E., Studies of acidosis. I. The bicarbonate concentration of the blood plasma; its significance, and its determination as a measure of acidosis. J. Biol. Chem., 1917, 30, 289.

II. A method for the determination of carbon dioxide and carbonates in solution. Ibid., 347.

9. Weech, A. A., Reeves, E. B., and Goettsch, E., The relationship between specific gravity and protein content in plasma, serum, and transudate from dogs. J. Biol. Chem., 1936, 113, 167.

10a. Barbour, H. G., and Hamilton, W. F., Blood specific gravity: its significance and a new method for its determination. Am. J. Physiol., 1924, 69, 654.

b. Idem, The falling drop method for determining specific gravity. J. Biol. Chem., 1926, 69, 625.

11. Newburgh, L. H., and others, Further experiences with the measurement of heat production from insensible loss of weight. J. Nutrition, 1937, 13, 203.

12. Benedict, F. G., The influence of inanition on metabolism. Carnegie Institution of Washington, D. C., 1907, Publ. No. 77, pp. 463-465.

13. Benedict, F. G., A study of prolonged fasting. Carnegie Institution of Washington, D. C., 1915, Publ. No. 203, pp. 401-407.

14. Peters, J. P., and Van Slyke, D. D., Quantitative Clinical Chemistry. Williams and Wilkins Co., Baltimore, 1937, p. 782.

15. Gamble, J. L., Personal communication.

16. Freyberg, R. H., and Grant, R. L., Loss of minerals through the skin of normal humans when sweating is avoided. J. Clin. Invest., 1937, 16, 729.

17. Gamble, J. L., Ross, G. S., and Tisdall, F. F., The metabolism of fixed base during fasting. J. Biol. Chem., 1923, 57, 633.

18. Darrow, D. C., and Yannet, H., Metabolic studies of the changes in body electrolyte and distribution of body water induced experimentally by deficit of extracellular electrolyte J. Clin. Invest., 1936, 15, 419. 
19. Freeman, N. E., Hemorrhage in relation to shock. Ann. Surg., 1935, 101, 484.

20. Scudder, J., Shock: blood studies as a guide to therapy. Lippincott, Philadelphia, 1940, pp. 68-74.

21. Drew, C. R., Scudder, J., and Papps, J., Controlled fluid therapy with hematocrit, specific gravity, and plasma protein determinations. Surg. Gynec., and Obst., 1940, 70, 859.

22. Elkinton, J. R., Gilmour, M. T., and Wolff, W. A., The control of water and electrolyte balance in surgical patients. Ann. Surg., 1939, 110, 1050.
23. Wiley, F. H., and Wiley, L. L., The inorganic salt balance during dehydration and recovery. J. Biol. Chem., 1933, 101, 83.

24. Dill, D. B., Life, Heat, and Altitude. Harvard Univ. Press, Cambridge, 1938, pp. 50-72.

25. Coller, F. A., and Maddock, W. G., A study of dehydration in humans. Ann. Surg., 1935, 102, 947.

26. Smith, H. W., and others, Glomerular dynamics in the normal human kidney. J. Clin. Invest., 1940, $19,751$. 